SGP-TR--111

DE88 012259

SGP-TR-111

\title{
Decline Curve Derivative Analysis for Homogeneous and Composite Reservoirs
}

\author{
Jay A. Demski
}

June 1987

Financial support was provided through the Stanford Geothermal Program under Department of Energy Contract No. DE-AS03-80SF11459 and by the Department of Petroleum Engineering, Stanford University

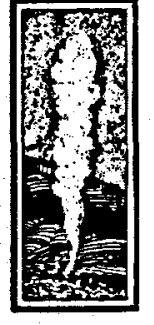

\section{Stanford Geothermal Program \\ Interdisciplinary Research in \\ Engineering and Earth Sciences \\ STANFORD UNIVERSITY \\ Stanford, California}

\section{DISCLAIMER}

\begin{abstract}
This report was prepared as an account of work sponsored by an agency of the United States Government. Neither the United States Government nor any agency thereof, nor any of their employees, makes any warranty, express or implied, or assumes any legal liability or responsibility for the accuracy, completeness, or usefulness of any information, apparatus, product, or process disclosed, or represents that its use would not infringe privately owned rights. Reference herein to any specific commercial product, process, or service by trade name, trademark, manufacturer, or otherwise does not necessarily constitute or imply its endorsement, recommendation, or favoring by the United States Government or any agency thereof. The views and opinions of authors expressed herein do not necessarily state or reflect those of the United States Government or any agency thereof.
\end{abstract}




\section{DISCLAIMER}

This report was prepared as an account of work sponsored by an agency of the United States Government. Neither the United States Government nor any agency Thereof, nor any of their employees, makes any warranty, express or implied, or assumes any legal liability or responsibility for the accuracy, completeness, or usefulness of any information, apparatus, product, or process disclosed, or represents that its use would not infringe privately owned rights. Reference herein to any specific commercial product, process, or service by trade name, trademark, manufacturer, or otherwise does not necessarily constitute or imply its endorsement, recommendation, or favoring by the United States Government or any agency thereof. The views and opinions of authors expressed herein do not necessarily state or reflect those of the United States Government or any agency thereof. 


\section{DISCLAIMER}

Portions of this document may be illegible in electronic image products. Images are produced from the best available original document. 


\section{ABSTRACT}

In this study, the rate decline and rate decline derivatives of a constant pressure well are presented for infinite, constant pressure outer boundary, and closed outer boundary homogeneous reservoirs. A rate derivative type curve is provided for these cases as well. The effects of the dimensionless reservoir exterior radius are discussed. Rate decline and rate decline derivatives of a constant pressure well in an infinite composite reservoir are also presented. For composite reservoirs, the effects of mobility ratios and discontinuity distance on both rate decline and rate decline derivatives are presented. Type curves for dimensionless wellbore flowrate derivatives for infinite composite reservoirs are provided. A new correlating group for the derivative type curve is provided, and is different than the correlating group for the rate type curve presented in the past. Finally, an analysis method that comprises type curve and derivative type curve matching to determine the dimensionless variables is proposed and demonstrated with a simulated example. 


\section{TABLE OF CONTENTS}

\section{$\underline{\text { Page }}$}

ABSTRACT

LIST OF FIGURES......................................................................................................iv

INTRODUCTION ...........................................................................................................

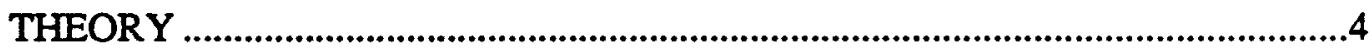

HOMOGENEOUS RESERVOIRS ........................................................................12

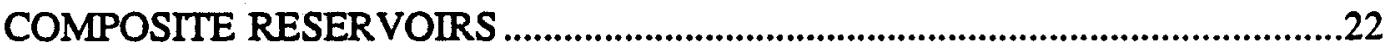

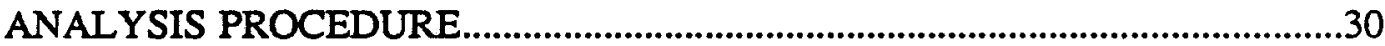

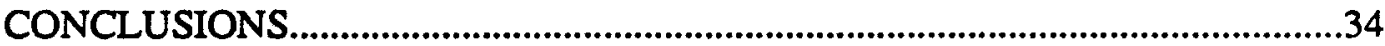

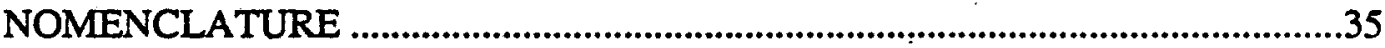

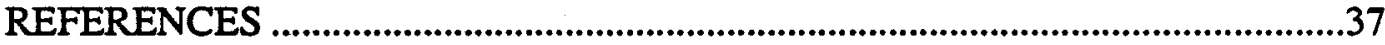




\section{LIST OF FIGURES}

Page

Figure 1. Composite reservoir configuration. (After Turki 1986).......................2

Figure 2. Rate decline curves for infinite, closed outer boundary, .....................13 and constant pressure outer boundary homogeneous reservoirs.

Log-log coordinates. $r_{e D}=50,100,200,500$.

Figure 3. Rate decline derivatives for infinite, closed outer boundary, 14 and constant pressure outer boundary homogeneous reservoirs.

Log-log coordinates. $r_{e D}=50,100,200,500$.

Figure 4. Rate decline curves for infinite, closed outer boundary, 15 and constant pressure outer boundary homogeneous reservoirs.

Semi-log coordinates. $r_{e D}=50,100,200,500$.

Figure 5. Rate decline derivatives for infinite, closed outer boundary, .16 and constant pressure outer boundary homogeneous reservoirs.

Semi-log coordinates. $r_{e D}=50,100,200,500$.

Figure 6. Fetkovich shifted rate declines. Closed and constant.

pressure outer boundaries, homogeneous reservoir.

$r_{e D}=50,500,5000$.

Figure 7. Fetkovich shifted $\log$-log rate derivatives. Closed and. constant pressure outer boundaries, homogeneous reservoir. $\mathrm{r}_{e D}=50,500,5000$.

Figure 8. Shifted (this study) $\log$-log rate derivatives. Closed and .21 constant pressure outer boundaries, homogeneous reservoir. $r_{e D}=50,500,5000$.

Figure 9. Effects of mobility ratio on rate decline. Composite reservoir, $R_{D}=50 . \lambda=0.1,0.5,1.0,5.0,10.0,50.0$.

Figure 10. Effects of mobility ratio on rate decline. Composite .24 reservoir, $R_{D}=500$. $\lambda=0.1,0.5,1.0,5.0,10.0,50.0$.

Figure 11. Fetkovich shifted composite reservoir rate declines. .26 . Composite reservoir, $R_{D}=50,500$. $\lambda=0.1,0.5,1.0$, $5.0,10.0,50.0$.

Figure 12. Effects of mobility ratio on $\log -\log$ rate derivatives. .27 Composite reservoir, $R_{D}=50$. $\lambda=0.1,0.5$, $1.0,5.0,10.0,50.0$. 
Figure 13. Effects of mobility ratio on $\log -\log$ rate derivatives.

Composite reservoir, $\mathbf{R}_{D}=500$. $\lambda=0.1,0.5$,

1.0, 5.0, 10.0, 50.0.

Figure 14. Shifted (this study) composite reservoir log-log.

rate derivatives. $R_{D}=50,100,500,1000$. $A: \lambda=50.0$,

B: $\lambda=10.0$, C: $\lambda=5.0, \mathrm{D}: \lambda=1.0, \mathrm{E}: \lambda=0.5$, F: $\lambda=0.1$.

Figure 15. Log-log match of rate data for a simulated example.

Figure 16. Log-log match of rate derivative data for a simulated example. 


\section{INTRODUCTION}

In the evaluation of petroleum and natural gas reserves, and in the evaluation of ground water aquifers, transient rate tests are performed. In these tests, the underground formation is produced at a constant pressure and the rate response at the active wellbore is measured.

Recent developments in the recording of flowrates with the use of spinners allows for the collection of very frequent rate measurements. Hence, the rate data is differentiable and a rate derivative response as a function of time can be constructed.

This study first concentrates on wellbore flowrate and flowrate derivative responses in three types of homogeneous reservoirs: infinite, constant pressure outer boundary, and closed outer boundary. Fetkovich (1980) examined decline rates of infinite and closed outer boundary reservoirs and developed a method of collapsing them into type curves. Next, flowrate derivative responses for infinite composite reservoirs are examined. The configuration of a composite reservoir is illustrated in Figure 1 (after Turki, 1986). A composite reservoir is defined as a system of two concentric regions with a single well at the center. The two reservoir regions have different properties (permeability, porosity, fluid viscosity, reservoir volume factor, and compressibility). An infinitesimally thin radial discontinuity is assumed to separate the two reservoir regions. Turki (1986) collapsed the flowrate responses for infinite composite reservoirs into type curves using the same method as Fetkovich (1980) and was able to determine the discontinuity distance and mobility ratio for certain conditions. In this way, Turki (1986) showed that graphed solutions for the dimensionless wellbore

flowrate in composite reservoirs exhibit at least two inflection points. Thus, if the flowrate derivative is graphed instead of the flowrate, these inflection points correspond 


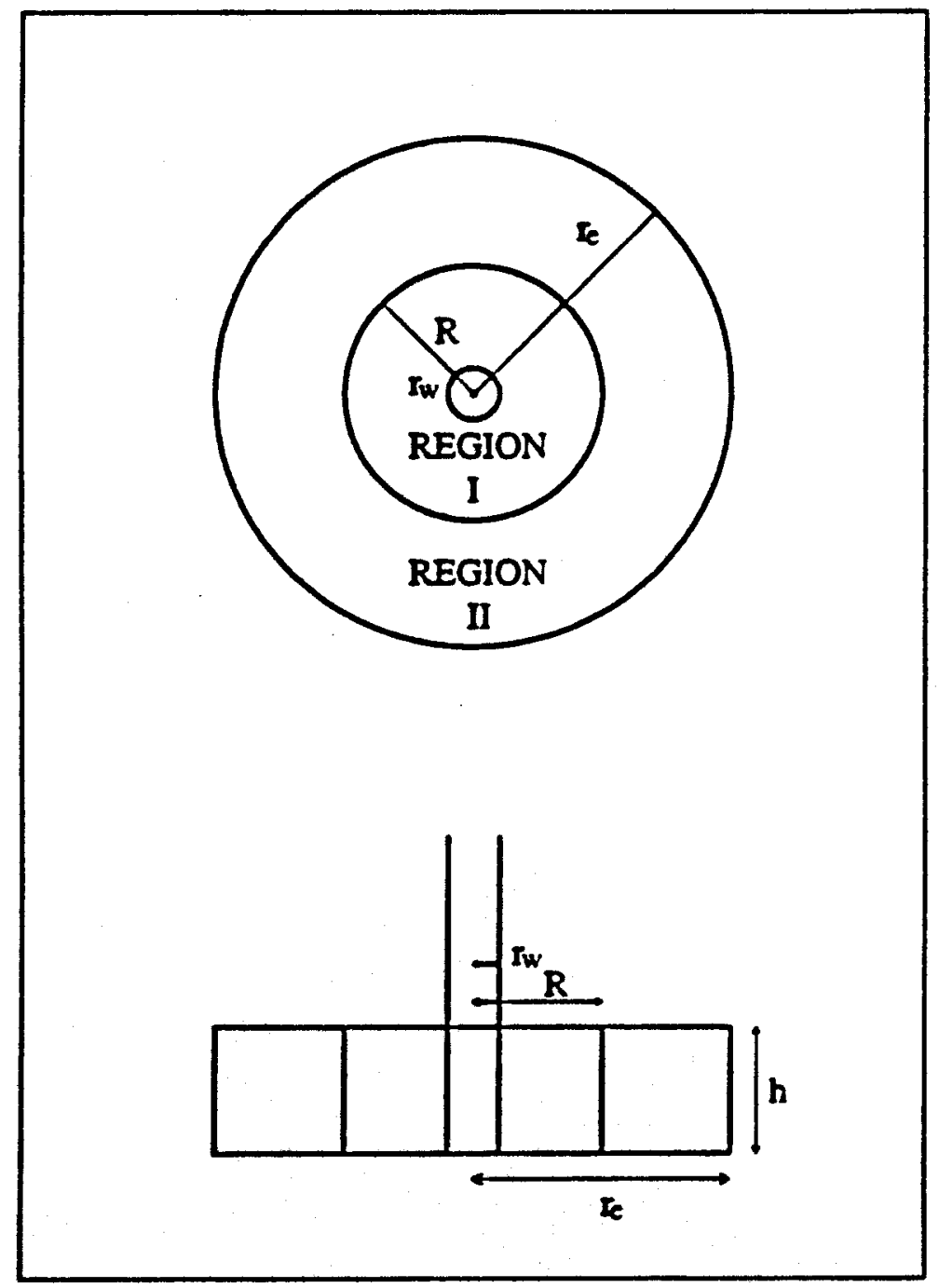

Figure 1. Composite reservoir configuration. (After Turki 1986) 
to either minima or maxima that can be easily identified. This study also examines the possibility of collapsing the rate derivative responses of infinite composite reservoirs. 


\section{THEORY}

The pressure responses in the two reservoir regions are described by two partial differential diffusivity equations. These equations are converted into a system of ordinary differential equations using the Laplace transformation. By applying the appropriate boundary conditions, Laplace space solutions for the transient rate decline in a composite reservoir are generated for infinite, closed outer boundary, and constant pressure outer boundary reservoirs. The inversion of the Laplace solutions to real space is performed numerically by an algorithm developed by Stehfest (1970). Here, the treatment of the theoretical background is modeled after the work of Turki (1986).

The usual assumptions of slightly compressible fluid, isotropic and homogeneous reservoir, and small pressure gradients everywhere are assumed in deriving the diffusivity equations in both reservoir regions. In addition, a few other assumptions are needed to solve the transient rate decline problem in the composite reservoir:

1. The formation is horizontal and of uniform thickness.

2. The discontinuity is of infinitesimal thickness in the radial direction.

3. Only single phase flow is considered. Furthermore, only one kind of fluid is considered in a given reservoir region.

4. During the testing period, the distance to the radial discontinuity is constant.

5. The sandface wellbore pressure is maintained constant throughout the testing period.

Fluid flow through both reservoir regions described in Figure 1 is idealized by the diffusivity equation. 
For Region I:

$$
\frac{\partial^{2} p_{1}}{\partial r^{2}}+\frac{1}{r} \frac{\partial p_{1}}{\partial r}=\left[\frac{\phi \mu c_{t}}{k}\right]_{1} \frac{\partial p_{1}}{\partial t} \quad r_{w}<r<R
$$

For Region II:

$$
\frac{\partial^{2} \mathrm{p}_{2}}{\partial^{2}}+\frac{1}{\mathrm{r}} \frac{\partial \mathrm{p}_{2}}{\partial \mathrm{r}}=\left(\frac{\phi \mu c_{\mathrm{t}}}{\mathrm{k}}\right)_{2} \frac{\partial \mathrm{p}_{2}}{\partial \mathrm{t}} \quad \mathrm{R}<\mathrm{r}<\infty
$$

where $R$ is the radial distance to the discontinuity.

The initial and inner boundary conditions are specified by the following equations:

$$
\begin{array}{cc}
p_{1}(r, 0)=p_{i} & r_{w}<r<R \\
p_{2}(r, 0)=p_{i} & R<r<\infty \\
p_{1}\left(r_{w}, t\right)=p_{w}
\end{array}
$$

Equations (3) and (4) specify that the entire reservoir is initially at a constant pressure, $\mathrm{p}_{\mathrm{i}}$. At the discontinuity, Equations (6) and (7) hold.

$$
\begin{gathered}
p_{1}(R, t)=p_{2}(R, t) \\
\frac{\partial p_{2}}{\partial r}=\lambda \frac{\partial p_{1}}{\partial r} \quad r=R \text { and } t>0
\end{gathered}
$$

where $\lambda$ is the mobility ratio to be defined later. To completely specify the problem, the appropriate outer boundary condition is needed. If the reservoir is infinite, the condition is:

$$
p_{2}(r, t) \rightarrow p_{i}, r \rightarrow \infty
$$

For a closed outer boundary reservoir, the condition is expressed as:

$$
\frac{\partial p_{2}}{\partial r}\left(r_{e}, t\right)=0 \quad R<r_{e}<\infty
$$


The condition for a constant pressure outer boundary is:

$$
p_{2}\left(r_{e}, t\right)=p_{i} \quad R<r_{e}<\infty
$$

Expressing all of the above equations in dimensionless variables results in the following set of equations:

$$
\begin{gathered}
\frac{\partial^{2} p_{D 1}}{\partial r_{D}^{2}}+\frac{1}{r_{D}} \frac{\partial p_{D 1}}{\partial r_{D}}=\frac{\partial p_{D 1}}{\partial t_{D}} \quad 1<r_{D}<R_{D} \\
\frac{\partial^{2} p_{D 2}}{\partial r_{D}^{2}}+\frac{1}{r_{D}} \frac{\partial p_{D 2}}{\partial r_{D}}=\eta \frac{\partial p_{D 2}}{\partial t_{D}} \quad R_{D}<r_{D}<\infty \\
p_{D 1}\left(r_{D}, 0\right)=0 \quad 1<r_{D}<R_{D} \\
p_{D 2}\left(r_{D}, 0\right)=0 \quad R_{D}<r_{D}<\infty \\
p_{D 1}\left(1, t_{D}\right)=1 \\
P_{D 1}\left(R_{D}, t_{D}\right)=p_{D 2}\left(R_{D}, t_{D}\right) \\
\frac{\partial p_{D 2}}{\partial r_{D}}=\lambda \frac{\partial p_{D 1}}{\partial r_{D}} \quad r_{D}=R_{D} \text { and } t_{D}>0
\end{gathered}
$$

For the infinite outer boundary case:

$$
\mathrm{PD}_{2}\left(\mathrm{r}_{\mathrm{D}}, \mathrm{t}_{\mathrm{D}}\right) \rightarrow 0, \quad, \mathrm{r}_{\mathrm{D}} \rightarrow \infty
$$

For the closed outer boundary case:

$$
\frac{\partial p_{D 2}}{\partial r_{D}}\left(r_{e D}, t_{D}\right)=0 \quad R_{D}<r_{e D}
$$

For the constant pressure outer boundary case:

$$
P_{D 2}\left(r_{C D}, t_{D}\right)=0 \quad R_{D}<r_{C D}
$$


The dimensionless variables are defined as follows:

$$
\begin{aligned}
& p_{D}=\frac{p_{i}-p}{p_{i}-p_{w}} \\
& t_{D}=t_{D 1}=\left[\frac{k}{\phi \mu c_{t} r_{w}{ }^{2}}\right]_{1} t \\
& \mathrm{r}_{\mathrm{D}}=\frac{\mathrm{r}}{\mathrm{r}_{\mathrm{w}}} \\
& R_{D}=\frac{R}{r_{w}} \\
& \lambda=\frac{\left[\frac{\mathrm{k}}{\mu}\right]_{1}}{\left[\frac{\mathrm{k}}{\mu}\right]_{2}} \\
& \eta=\frac{\left(\frac{\mathrm{k}}{\phi \mu c_{\mathrm{t}}}\right)_{1}}{\left(\frac{\mathrm{k}}{\phi \mu c_{\mathrm{t}}}\right)_{2}}=\lambda \frac{\left(\phi c_{\mathrm{t}}\right)_{2}}{\left(\phi c_{\mathrm{t}}\right]_{1}}
\end{aligned}
$$

From Darcy's equation, the relationship between the flow rate and the pressure at any location within the composite reservoir is derived.

For Region I:

$$
q(r, t)_{1}=\left[\frac{2 \pi k h}{\mu}\right]_{1} r \frac{\partial p_{1}}{\partial r}, \quad r_{w} \leq r \leq R
$$

For Region II:

$$
\mathrm{q}(\mathrm{r}, \mathrm{t})_{2}=\left[\frac{2 \pi k h}{\mu}\right]_{2} \mathrm{r} \frac{\partial \mathrm{p}_{2}}{\partial \mathrm{r}}, \quad \mathrm{R} \leq \mathrm{r}<\infty
$$


In terms of dimensionless variables, Equations (27) and (28) can be expressed as:

$$
\begin{array}{cc}
q_{D 1}=-r_{D} \frac{\partial p_{D 1}}{\partial r_{D}}, & 1 \leq r_{D} \leq R_{D} \\
q_{D 2}=-\frac{1}{\lambda} r_{D} \frac{\partial p_{D 2}}{\partial r_{D}}, & R_{D} \leq r_{D}<\infty
\end{array}
$$

where the dimensionless flow rate is defined in the following manner:

$$
q_{D}=\frac{\mu_{1} q(r, t)}{k_{1} h\left(p_{i}-p_{w}\right)}
$$

Applying the Laplace transformation to the partial differential Equations (11) and (12), and using the initial conditions of Equations (13) and (14) yields a set of ordinary differential equations:

$$
\begin{array}{ll}
\frac{d^{2} \bar{p}_{D 1}}{d r_{D}{ }^{2}}+\frac{1}{r_{D}} \frac{d \bar{p}_{D 1}}{d r_{D}}=s \bar{p}_{D 1}, & 1<r_{d}<R_{D} \\
\frac{d^{2} \bar{p}_{D 2}}{d r_{D}{ }^{2}}+\frac{1}{r_{D}} \frac{d \bar{p}_{D 2}}{d r_{D}}=\eta s \bar{p}_{D 2}, & R_{D}<r_{D}<\infty
\end{array}
$$

Equations (32) and (33) are simple forms of modified Bessel equations (Abramowitz and Stegun pp 374, 1964). The general solutions to the equations where the Laplace transformation of the pressure, $\overline{\mathrm{P}}_{\mathrm{D}}$, is a function of the Laplace variable, $\mathrm{s}$, and the spatial variable, $\mathrm{r}_{\mathrm{D}}$, are:

$$
\begin{array}{cl}
\overline{\mathrm{P}}_{D 1}=C_{11} \mathrm{I}_{0}\left(\mathrm{r}_{D} \sqrt{s}\right)+C_{12} \mathrm{~K}_{0}\left(\mathrm{r}_{D} \sqrt{s}\right), & 1<\mathrm{r}_{D}<R_{D} \\
\overline{\mathrm{P}}_{\mathrm{D} 2}=C_{21} \mathrm{I}_{0}\left(\mathrm{r}_{D} \sqrt{\eta s}\right)+C_{22} \mathrm{~K}_{0}\left(\mathrm{r}_{D} \sqrt{\eta s}\right), & \mathrm{R}_{\mathrm{D}}<\mathrm{r}_{D}<\infty
\end{array}
$$

The flow in any of the two reservoir regions can be obtained by using either Equation (36) or Equation (37) which are respectively the Laplace transformations of Equations (29) and (30). 


$$
\begin{array}{cc}
\bar{q}_{D 1}=-r_{D} \frac{d \bar{p}_{D 1}}{d r_{D}}, \quad 1 \leq r_{D} \leq R_{D} \\
\bar{q}_{D 2}=-\frac{1}{\lambda} r_{D} \frac{d \bar{p}_{D 2}}{d r_{D}}, \quad . \quad R_{D} \leq r_{D}<\infty
\end{array}
$$

To determine the constants $\mathrm{C}_{11}, \mathrm{C}_{12}, \mathrm{C}_{21}$, and $\mathrm{C}_{22}$, the appropriate boundary conditions are used in addition to the conditions at the discontinuity. In Laplace space, Equations (15) through (20) become, respectively:

$$
\begin{aligned}
& \overline{\mathrm{p}}_{\mathrm{D} 1}(1, \mathrm{~s})=\frac{1}{\mathrm{~s}} \\
& \overline{\mathrm{p}}_{\mathrm{D} 1}\left(\mathrm{R}_{\mathrm{D}}, \mathrm{s}\right)=\overline{\mathrm{p}}_{\mathrm{D} 2}\left(\mathrm{R}_{\mathrm{D}}, \mathrm{s}\right) \\
& \frac{d \bar{p}_{D 2}}{d r_{D}}=\lambda \frac{d \bar{p}_{D 1}}{d r_{D}}, \quad r_{D}=R_{D} \text { and } t_{D}>0 \\
& \overline{\mathrm{p}}_{\mathrm{D} 2}\left(\mathrm{r}_{\mathrm{D}}, \mathrm{s}\right) \rightarrow 0, \mathrm{r}_{\mathrm{D}} \rightarrow \infty \\
& \frac{d \bar{p}_{D 2}}{d r_{D}}\left(r_{e D}, s\right)=0, \quad R_{D}<r_{e D}<\infty \\
& \overline{\mathrm{p}}_{\mathrm{D} 2}\left(\mathrm{r}_{\mathrm{eD}}, \mathrm{s}\right)=0, \quad \mathrm{R}_{\mathrm{D}}<\mathrm{r}_{\mathrm{eD}}<\infty
\end{aligned}
$$

For instance, the constants for the infinite reservoir case are obtained by using Equations (38), (39), (40), and (41). At the wellbore, $\mathrm{r}_{D}=1$, and:

$$
\bar{q}_{D 1}=-\sqrt{s}\left[C_{11} I_{1}(\sqrt{s})-C_{12} K_{1}(\sqrt{s})\right]
$$

In region II the flowrate is:

$$
\bar{q}_{D 2}=C_{22} r_{D} \sqrt{\eta s} K_{1}\left(r_{D} \sqrt{\eta s}\right), \quad R_{D}<r_{D}<\infty
$$


The constants $C_{11}, C_{12}$, and $C_{22}$ have to satisfy the following system of linear equations:

$$
\begin{gathered}
C_{11} I_{0}(\sqrt{s})+C_{12} K_{0}(\sqrt{s})=\frac{1}{s} \\
I_{0}\left(R_{D} \sqrt{s}\right) C_{11}+K_{0}\left(R_{D} \sqrt{s}\right) C_{12}-K_{0}\left(R_{d} \sqrt{\eta s}\right) C_{22}=0 \\
\lambda I_{1}\left(R_{D} \sqrt{s}\right) C_{11}-\lambda K_{1}\left(R_{D} \sqrt{s}\right) C_{12}+\sqrt{\eta} K_{1}\left(R_{D} \sqrt{\eta s}\right) C_{22}=0
\end{gathered}
$$

This system of linear equations is solved by direct substitution to yield:

$$
C_{11}=\frac{1}{s D}\left[\lambda K_{1}\left(R_{D} \sqrt{s}\right) K_{0}\left(R_{D} \sqrt{\eta s}\right)-\sqrt{\eta} K_{0}\left(R_{D} \sqrt{s}\right) K_{1}\left(R_{D} \sqrt{\eta s}\right)\right]
$$

where

$$
\begin{gathered}
D=\left[\lambda I_{1}\left(R_{D} \sqrt{s}\right) K_{0}\left(R_{D} \sqrt{\eta s}\right)+\sqrt{\eta} I_{0}\left(R_{D} \sqrt{s}\right) K_{1}\left(R_{D} \sqrt{\eta s}\right)\right] K_{0}(\sqrt{s}) \\
+\left[\lambda K_{1}\left(R_{D} \sqrt{s}\right) K_{0}\left(R_{D} \sqrt{\eta s}\right)-\sqrt{\eta} K_{0}\left(R_{D} \sqrt{s}\right) K_{1}\left(R_{D} \sqrt{\eta s}\right)\right] I_{0}(\sqrt{s}) \\
C_{12}=\frac{1-s\left[I_{0}(\sqrt{s})-s \sqrt{s} I_{1}(\sqrt{s})\right] C_{11}}{s\left[K_{0}(\sqrt{s})+S \sqrt{s} K_{1}(\sqrt{s})\right]} \\
C_{22}=\frac{K_{0}\left(R_{D} \sqrt{s}\right)+s\left\{\left[K_{0}(\sqrt{s})+S \sqrt{s} K_{1}(\sqrt{s})\right] I_{0}\left(R_{D} \sqrt{s}\right)-\left[I_{0}(\sqrt{s})-S \sqrt{s} I_{1}(\sqrt{s})\right] K_{0}\left(R_{D} \sqrt{s}\right)\right\} C_{11}}{s K_{0}\left(R_{D} \sqrt{\eta s}\right)\left[K_{0}(\sqrt{s})+S \sqrt{s} K_{1}(\sqrt{s})\right]}
\end{gathered}
$$

The inversion of the solution from Laplace space to real space is performed numerically by an algorithm developed by Stehfest (1970). 
The homogeneous Laplace space rate solutions are as follows (after Fetkovich, 1980):

Infinite Case:

$$
\bar{q}_{D}=\frac{\sqrt{s K_{1}}(\sqrt{s})}{s K_{0}(\sqrt{s})}
$$

Closed Finite Systems:

$$
\bar{q}_{D}=\frac{\sqrt{s}}{s} \frac{\left[I_{1}\left(r_{e D} \sqrt{s}\right) K_{1}(\sqrt{s})-K_{1}\left(r_{e D} \sqrt{s}\right) I_{1}(\sqrt{s})\right]}{\left[K_{1}\left(r_{e D} \sqrt{s}\right) I_{0}(\sqrt{s})+I_{1}\left(r_{e D} \sqrt{s}\right) K_{0}(\sqrt{s})\right]}
$$

Constant Pressure Finite Systems:

$$
\bar{q}_{D}=\frac{\sqrt{s}}{s} \frac{\left[I_{0}\left(r_{e D} \sqrt{s}\right) K_{1}(\sqrt{s})+K_{0}\left(r_{e D} \sqrt{s}\right) I_{1}(\sqrt{s})\right]}{\left[K_{1}\left(r_{e D} \sqrt{s}\right) I_{0}(\sqrt{s})+I_{1}\left(r_{e D} \sqrt{s}\right) K_{0}(\sqrt{s})\right]}
$$

Finally, since the rate at $t=0$ is zero, we have:

$$
L\left\{\frac{\partial q_{D}}{\partial t_{D}}\right\}=s \bar{q}_{D}
$$

Hence, for the $\log$-log derivative, equation (57) is used:

$$
\frac{\partial \ln \left(q_{D}\right)}{\partial \ln \left(t_{D}\right)}=\frac{t_{D}}{q_{D}} \frac{\partial q_{D}}{\partial t_{D}}=\frac{t_{D}}{q_{D}} L^{-1}\left\{s \bar{q}_{D}\right\}
$$

For the semi-log derivative, equation (58) is used:

$$
\frac{\partial q_{D}}{\partial \ln t_{D}}=t_{D} L^{-1}\left\{s \bar{q}_{D}\right\}
$$




\section{HOMOGENEOUS RESERVOIRS}

Figure 2 presents the dimensionless wellbore flow rate declines for homogeneous reservoirs. Three types of reservoirs are considered: infinite, constant pressure outer boundary, and closed outer boundary. The exterior radii are $r_{e D}=50,100,200$, and 500. The rate decline for the closed outer boundary cases were considered by Fetkovich (1980). At early time, the rate declines are all infinite acting. Only when the effects of different $\mathrm{r}_{\mathrm{eD}}$ 's are encountered do the rates deviate from the infinite case, and the deviation for the two different outer boundary conditions occur at the same time. Fetkovich (1980) also observed that the rate decline for a closed outer boundary reservoir is exponential. The constant pressure rate responses become almost constant at late time as the flow approaches a steady state condition.

Log-log decline curve derivatives for the same cases as presented in Figure 2 are shown in Figure 3 in log-log coordinates. Here again, the time of deviation from the infinite case depends on $r_{e D}$. The larger the $r_{e D}$, the later the deviation occurs. However, the deviation of the rate decline derivatives from the infinite case derivative occur almost one log cycle earlier than the corresponding deviation observed in the rate decline. The constant pressure rate derivatives approach zero as the constant pressure rates approach constant values for differing $r_{e D}$ 's. The closed outer boundary derivatives increase in a linear fashion on the log-log graph and become a straight line at late time with a slope of unity.

The same decline rates in Figure 2 are presented on semi-log coordinates in Figure 4. The corresponding semi-log rate derivatives are shown in Figure 5 in semi-log coordinates. Here again the deviations of the derivatives from the infinite case derivative occurs earlier than in the rate decline Figure for the same data. The very apparent maxima and minima in the semi-log derivatives are reflections of the inflection points 


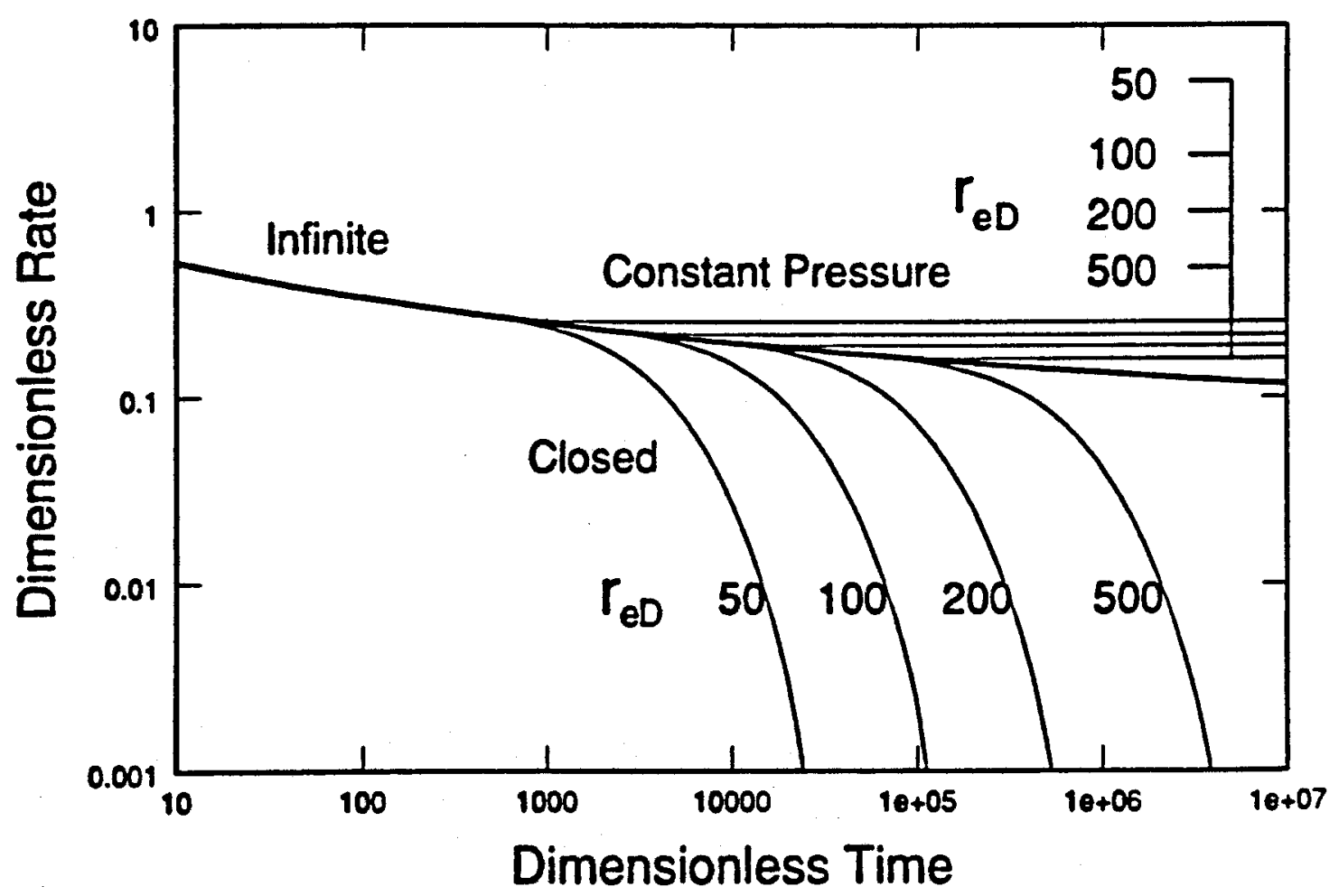

Figure 2. Rate decline curves for infinite, closed outer boundary, and constant pressure outer boundary homogeneous reservoirs. Log- $\log$ coordinates. $r_{e D}=50,100$, 200,500 . 


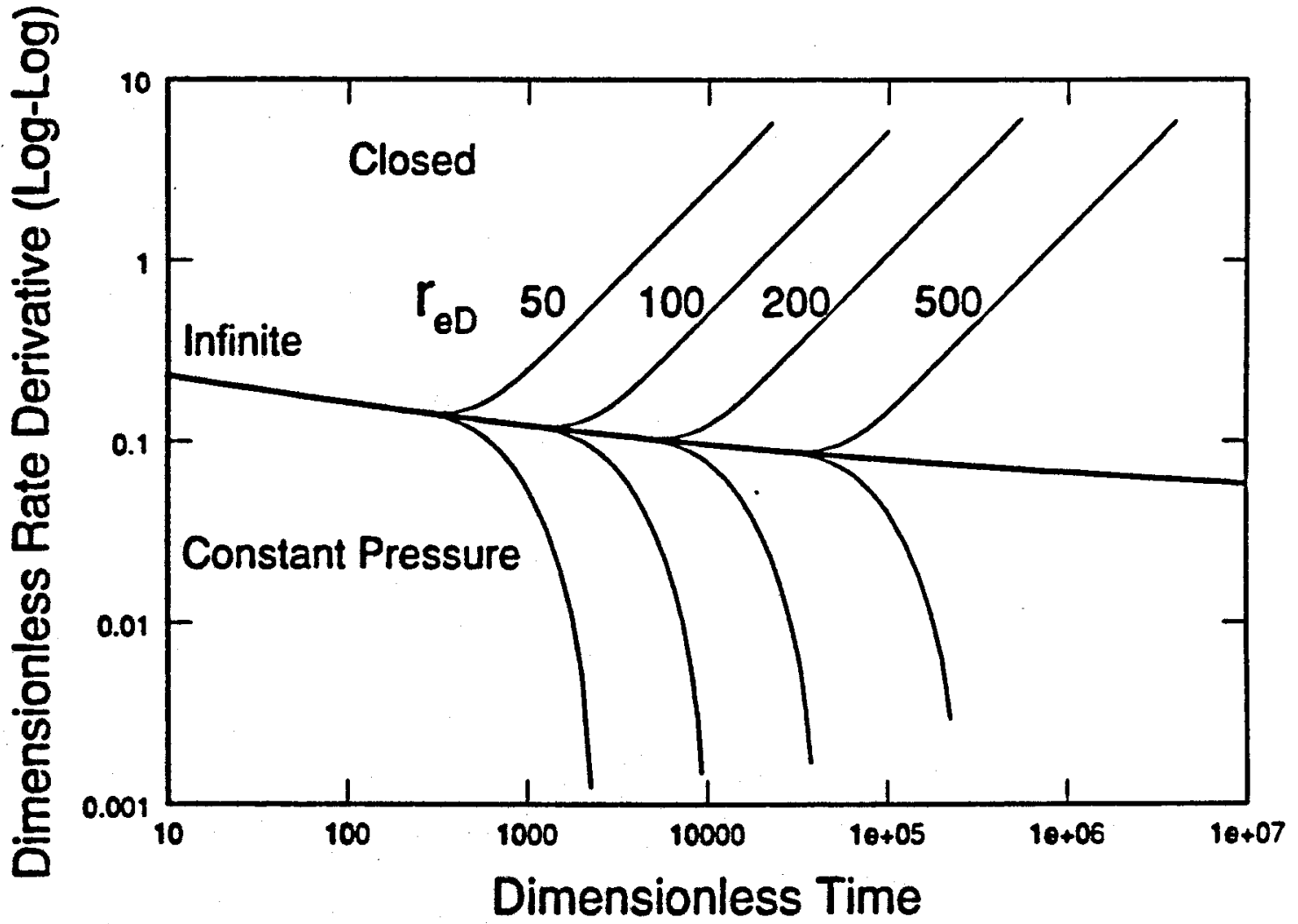

Figure 3. Rate decline derivatives for infinite, closed outer boundary, and constant pressure outer boundary homogeneous reservoirs. Log-log coordinates. $r_{e D}=50,100,200,500$. 


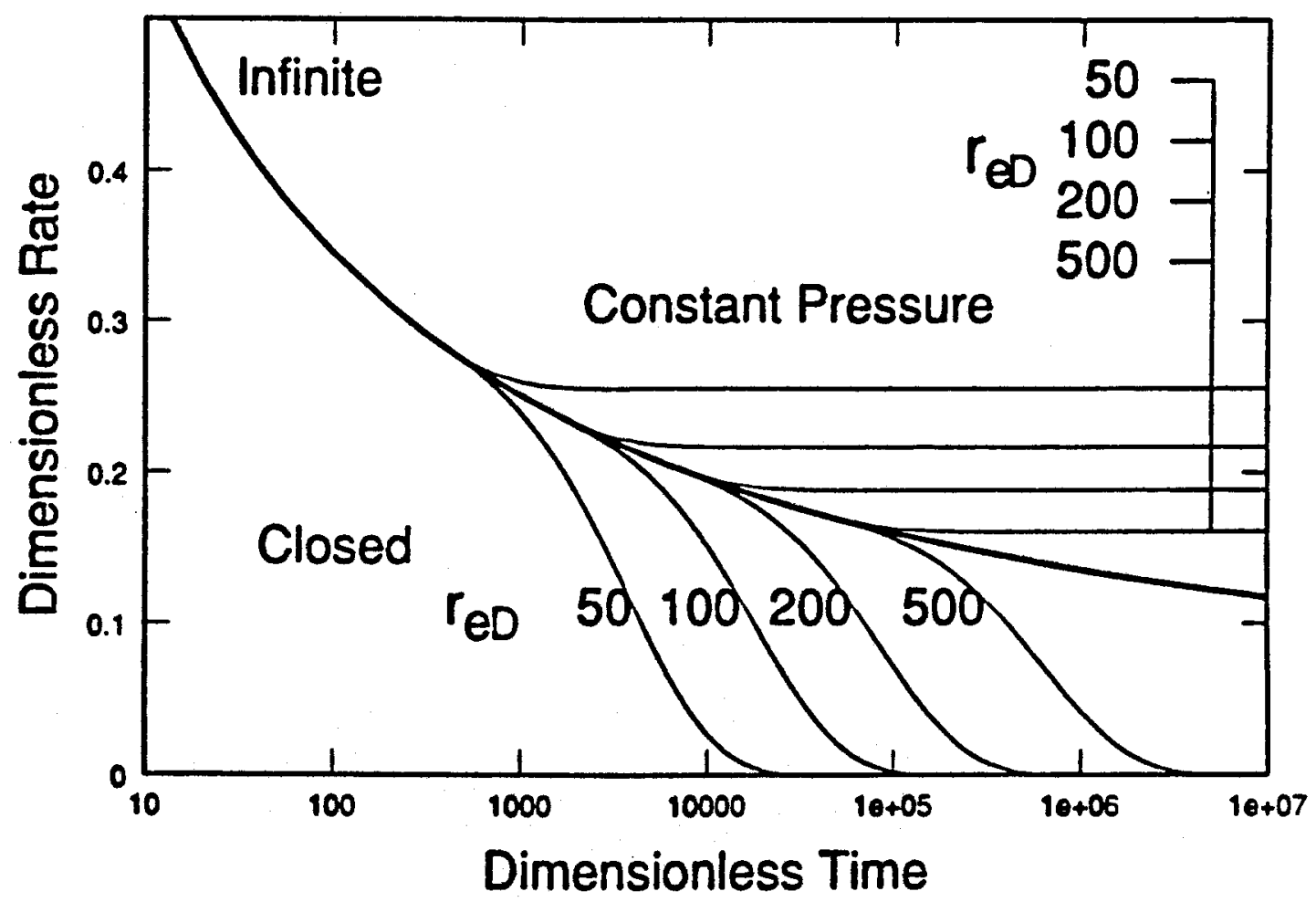

Figure 4. Rate decline curves for infinite, closed outer boundary, and constant pressure outer boundary homogeneous reservoirs. Semi-log coordinates. $r_{e}=50,100$, $200,500$. 


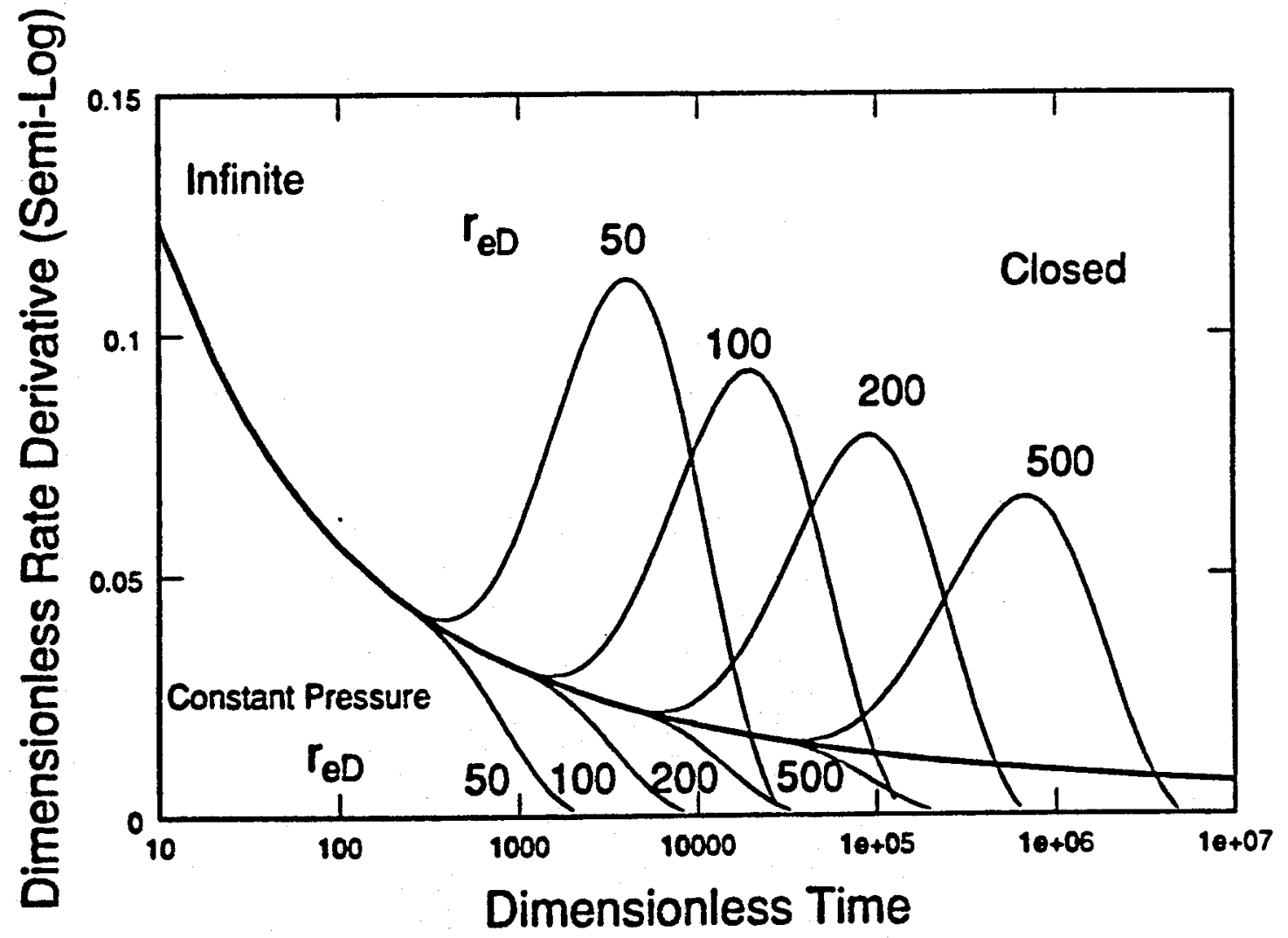

Figure 5. Rate decline derivatives for infinite, closed outer boundary, and constant pressure outer boundary homogeneous reservoirs. Semi-log coordinates. $\mathrm{r}_{e D}=50,100,200,500$. 
present in the semi-log rate declines. The derivatives for the constant pressure case deviate below the infinite acting derivatives and approach zero. The derivatives for the closed outer boundary case display significant character. The first inflection point in the semi-log rate decline (Figure 4) corresponds to a minimum in the semi-log derivative, and the derivative increases above the infinite acting derivative. The second inflection point in the rate response corresponds to a maximum value of the semi-log derivative. After this maximum, the semi-log derivative decreases rapidly and approaches zero as the reservoir depletes and reservoir pressure approaches the wellbore pressure.

Fetkovich (1980) presented a method for collapsing rate decline data into a type curve defining:

$$
t_{D d}=t_{D} \frac{1}{\frac{1}{2}\left[r_{e D}^{2}-1\right]\left[\ln \left(r_{e D}\right)-\frac{1}{2}\right]}
$$

and

$$
q_{D d}=q_{D}\left[\ln \left(r_{C D}\right)-\frac{1}{2}\right]
$$

In this collapsed curve, all solutions for various $r_{E D}$ 's in a closed outer boundary homogeneous reservoir converge to a single curve at the onset of depletion. This observation is shown in Figure 6 for $\mathrm{r}_{\mathrm{eD}}=50,500$, and 5000. The rate declines for different values of $r_{e D}$ in a constant pressure outer boundary homogeneous reservoir collapse into a single curve in the same manner (see Figure 6). Due to similarity in shape of the rate derivative curves for different $r_{e D}$ 's, it seems that they too would be collapsible into a single curve. The derivatives for the same cases as in Figure 6 are 


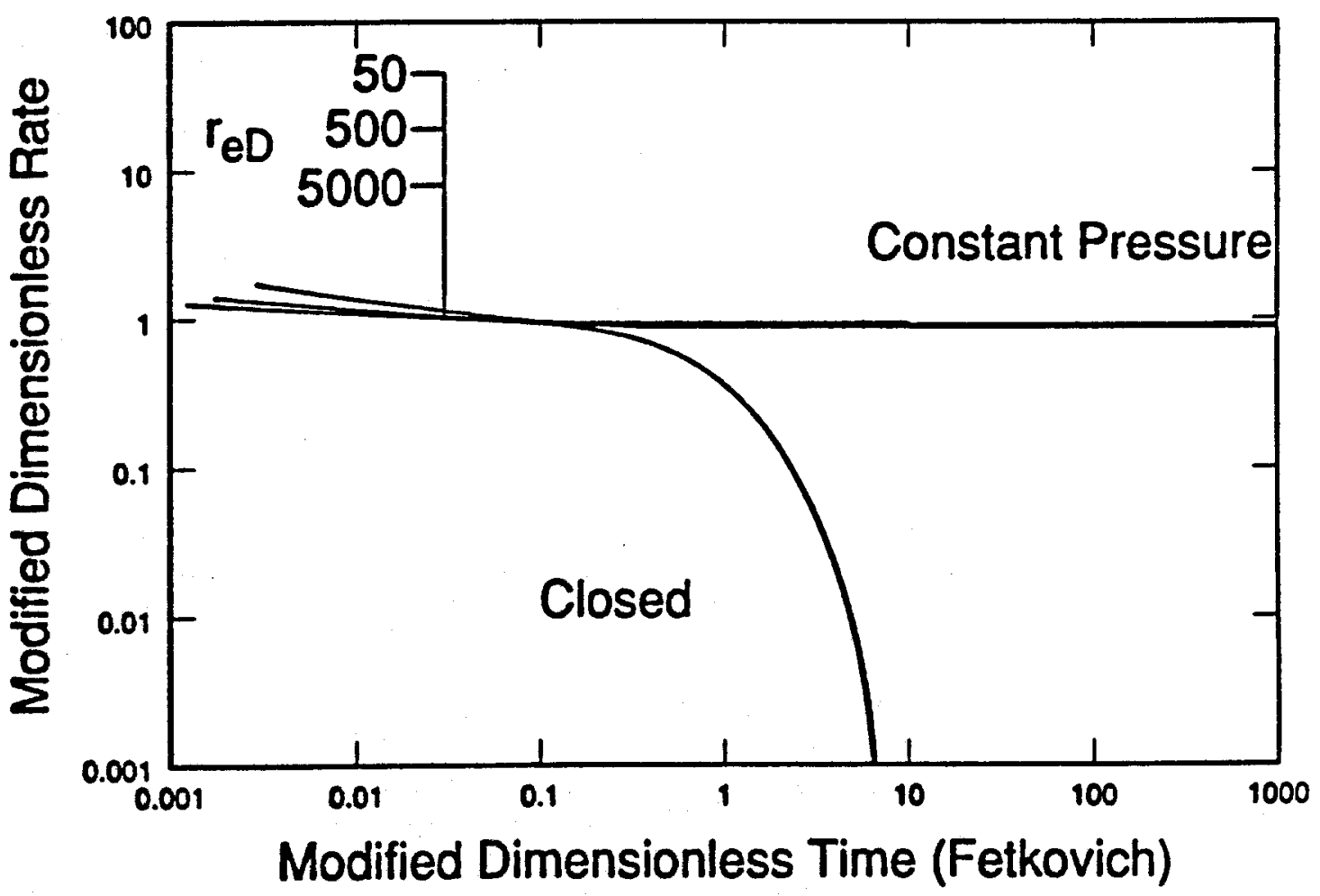

Figure 6. Fetkovich shifted rate declines. Closed and constant pressure outer boundaries, homogeneous reservoir. $r_{e D}=50,500,5000$. 
shown in Figure 7 collapsed together using Fetkovich's method. These curves do not shift into a single curve. However, if Fetkovich's dimensionless time is simplified to be:

$$
t_{D d}=t_{D} \frac{1}{\left[r_{e D}^{2}-1\right]}
$$

the constant pressure rate derivatives and the closed outer boundary derivatives collapse into individual curves, shown in Figure 8. The collapsing of the derivative curves for the constant pressure cases is not as good as the collapsing for the closed outer boundary case. However, for practial purposes, we can consider the curves to be collapsed. 


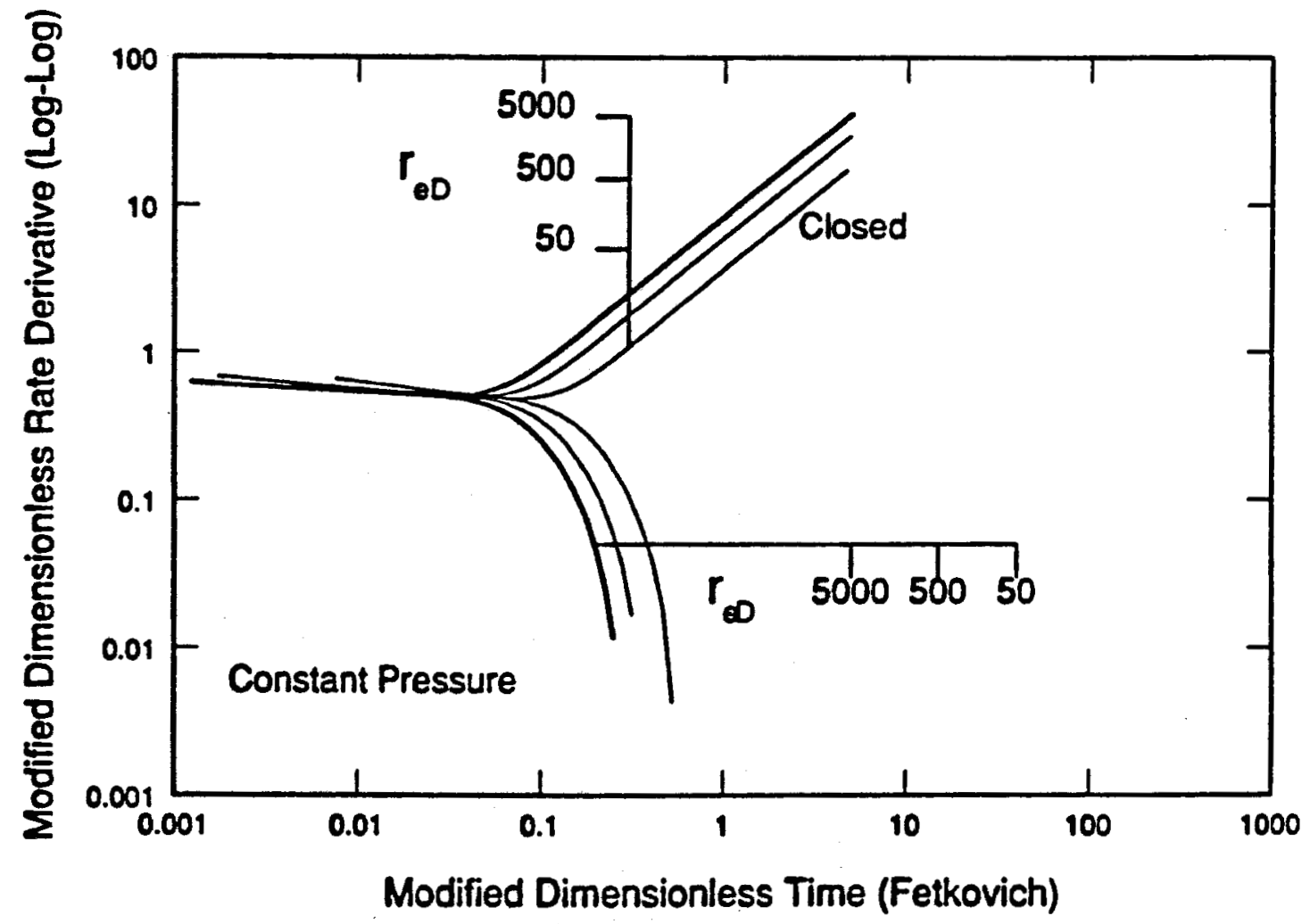

Figure 7. Fetkovich shifted $\log -\log$ rate derivatives. Closed and constant pressure outer boundaries, homogeneous reservoir. $r_{e D}=50,500,5000$. 


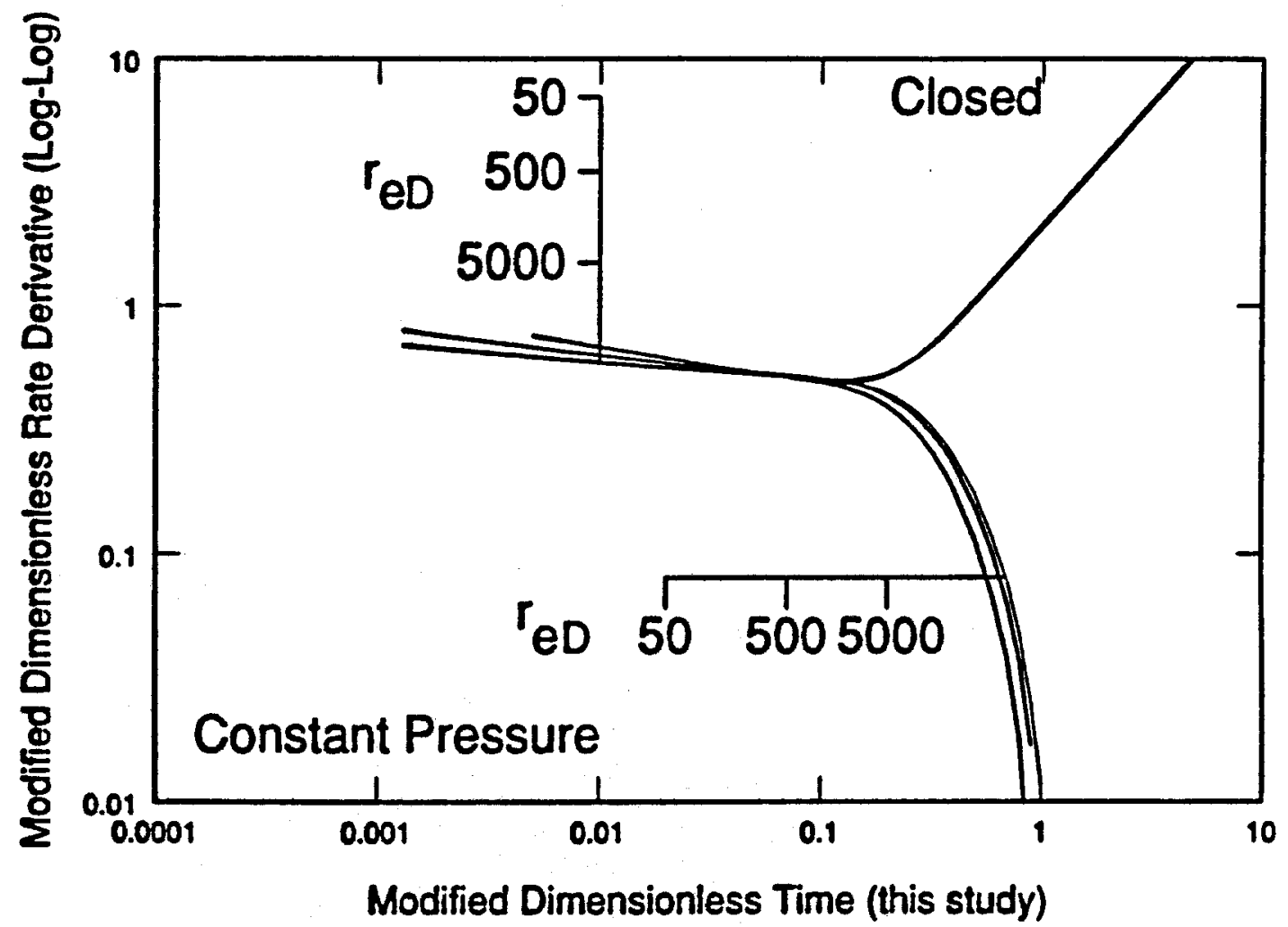

Figure 8. Shifted (this study) $\log -\log$ rate derivatives. Closed and constant pressure outer boundaries, homogeneous reservoir. $r_{e D}=50,500,5000$. 


\section{COMPOSITE RESERVOIRS}

Now let us turn our attention to infinite composite reservoir systems. Figure 9 presents rate decline data for a composite reservoir with $R_{D}=50$, and mobility ratios of $0.1,0.5,1.0,5.0,10.0$, and 50.0. At early time, the system behaves like an infinite homogeneous reservoir; the wellbore flow rate is independent of the mobility ratio and the rate decline is only a function of the properties of the inner region. The first infinite acting flow period is followed by a transition where the discontinuity radius begins to affect the wellbore flow rate response. If the mobility ratio is favorable, (the mobility of the outer region is larger than the mobility of the inner region) the transition corresponds to the transition observed in constant pressure outer boundary homogeneous reservoirs. If the mobility ratio is unfavorable, a portion of the transition flow period follows an exponential decline which is characteristic of a closed outer boundary reservoir. However, in the second infinite acting flow period, the wellbore flow rate response is a function of both the mobility ratio and the outer region properties. For favorable mobility ratios the transition flow period appears to be short and hardly noticeable due to the $\log -\log$ coordinates. For very favorable mobility ratios such as $\lambda=0.1$, the system behaves like a constant pressure outer boundary homogeneous reservoir. Turki (1986) showed that for the unfavorable mobility ratios, the exponential decline portion is noticeable only if $\lambda>10$, and that for mobility ratios between 5 and 10, only the transition flow period is apparent. This transition flow period becomes very short for mobility ratios less than 5 .

The dimensionless wellbore flow rate responses for an infinite composite reservoir with $R_{D}=500$ and mobility ratios of $0.1,0.5,1.0,5.0,10.0$, and 50.0 are presented in Figure 10. The curves are very similar in shape to the previous case with $R_{D}=50$, but the deviations from the infinite acting case occur approximately two log cycles later. 


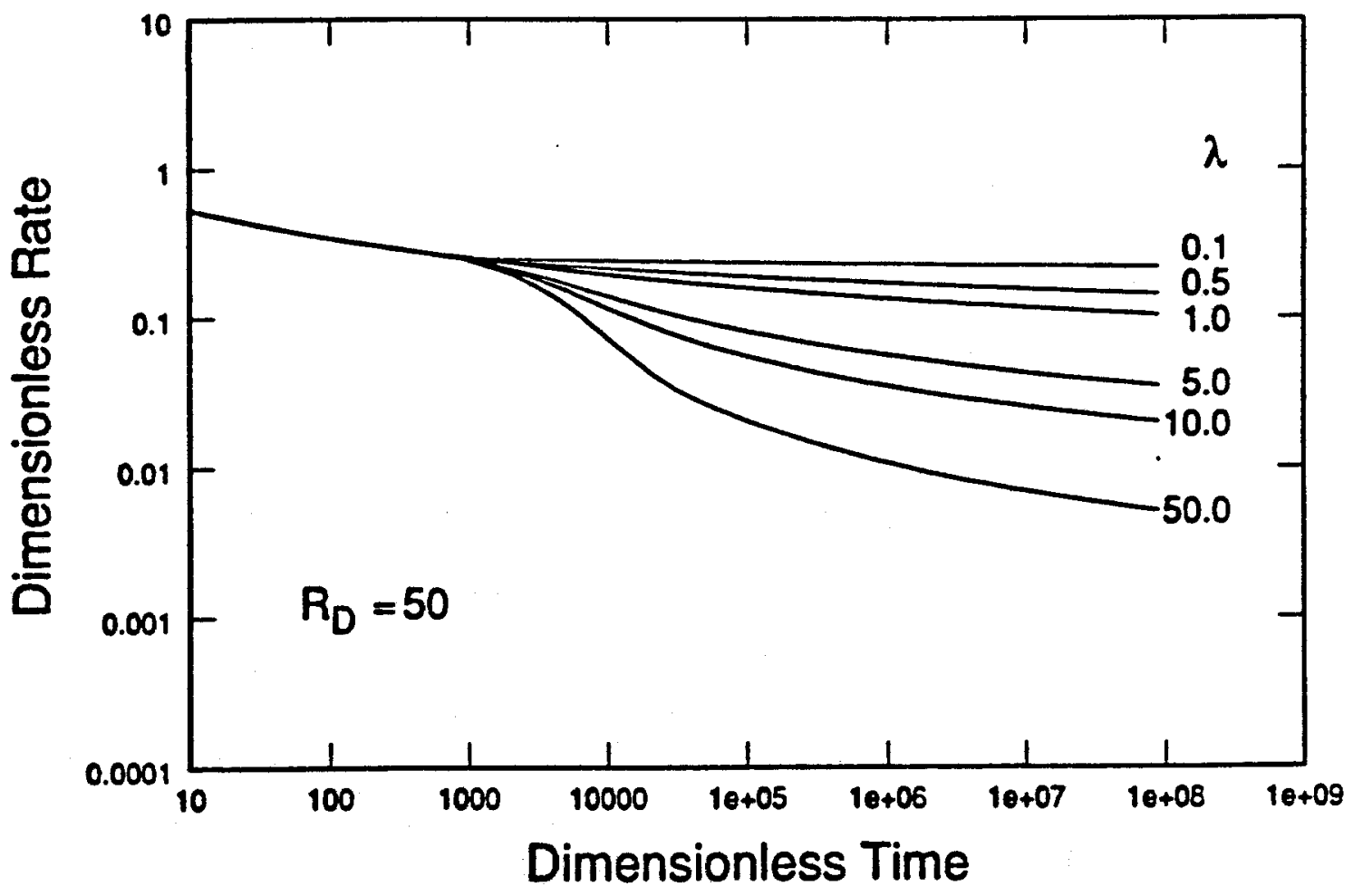

Figure 9. Effects of mobility ratio on rate decline. Composite reservoir, $R_{D}=50$. $\lambda=0.1,0.5,1.0,5.0,10.0,50.0$. 


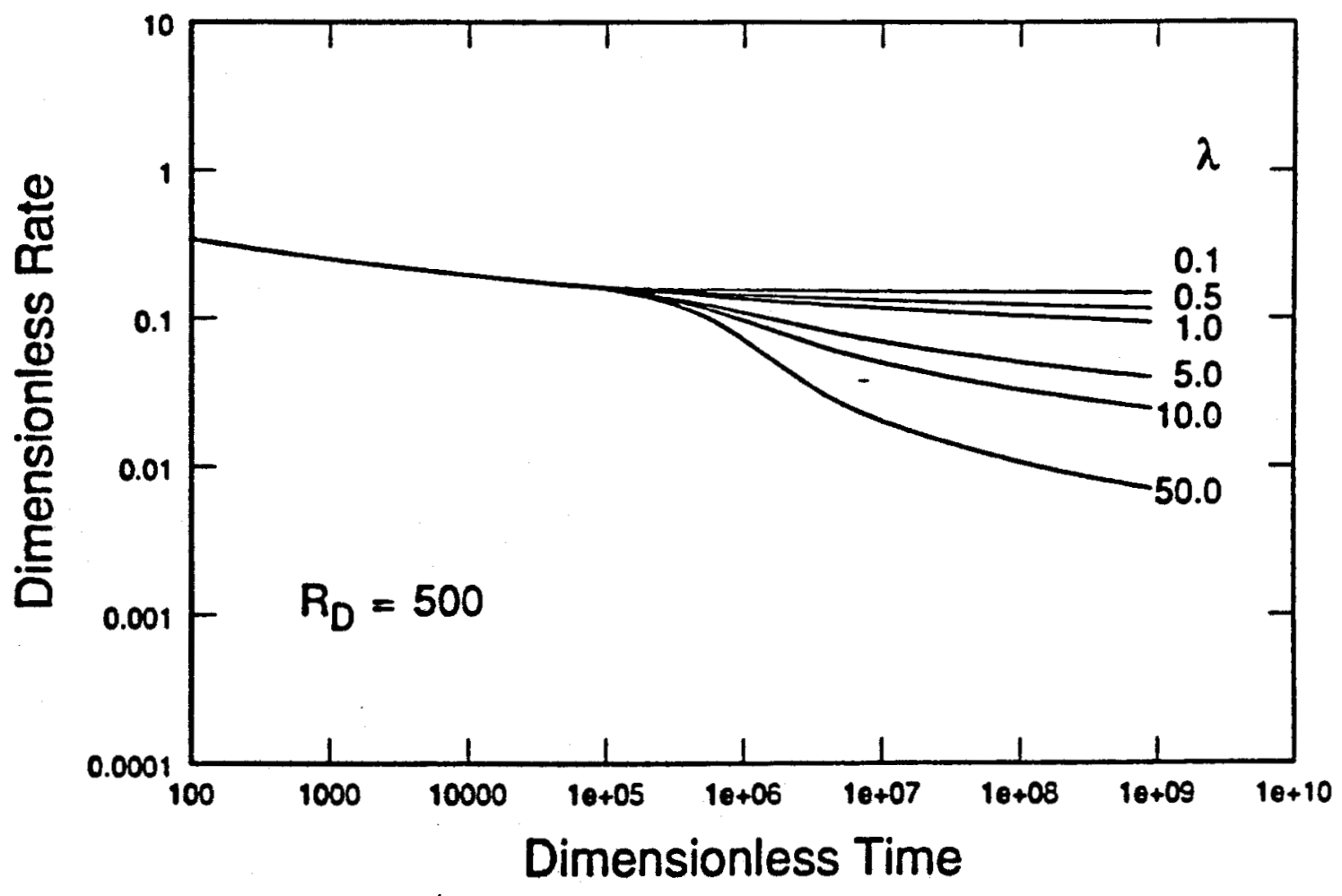

Figure 10. Effects of mobility ratio on rate decline. Composite reservoir, $R_{D}=500$. $\lambda=0.1,0.5,1.0,5.0,10.0,50.0$. 
Due to this similarity in shape, it would seem that the curves would be collapsible into a type curve, with the transition flow periods for different mobility ratios collapsing into single curves. Using Fetkovich's method again, Figure 11 shows that this is indeed the case, as was shown by Turki (1986). The match is fairly good, especially in the early portions of the transition flow periods for the different mobility ratios. This method for collapsing the rate responses of composite systems was presented by Turki (1986).

The $\log -\log$ derivatives of the wellbore flowrate response of composite systems with $R_{D}=50$ and 500 presented in Figures 9 and 10 are shown in Figures 12 and 13, respectively. The maxima and minima apparent in the derivative Figures correspond to inflection points present in the rate decline curves of Figures 9 and 10. Here, the deviations from the infinite acting portion of the derivative curve due to different mobility ratios occur earlier than the corresponding deviations present in the rate decline curves. The shapes of the derivative curves for various mobility ratios with $R_{D}$ $=50$ and 500 are very similar. This would lead us to believe that they too may be collapsible into single curves for the transition flow periods and various values of $R_{D}$. Using the modified "shifting" method developed for the homogeneous cases results in a very good match. This is shown in Figure 14. Again, the match is extremely good for the early transition flow periods for different mobility ratios. At later time, the match deteriorates, more noticeably for unfavorable mobility ratios, but the earlier time transition period data is most important in well test analysis. 


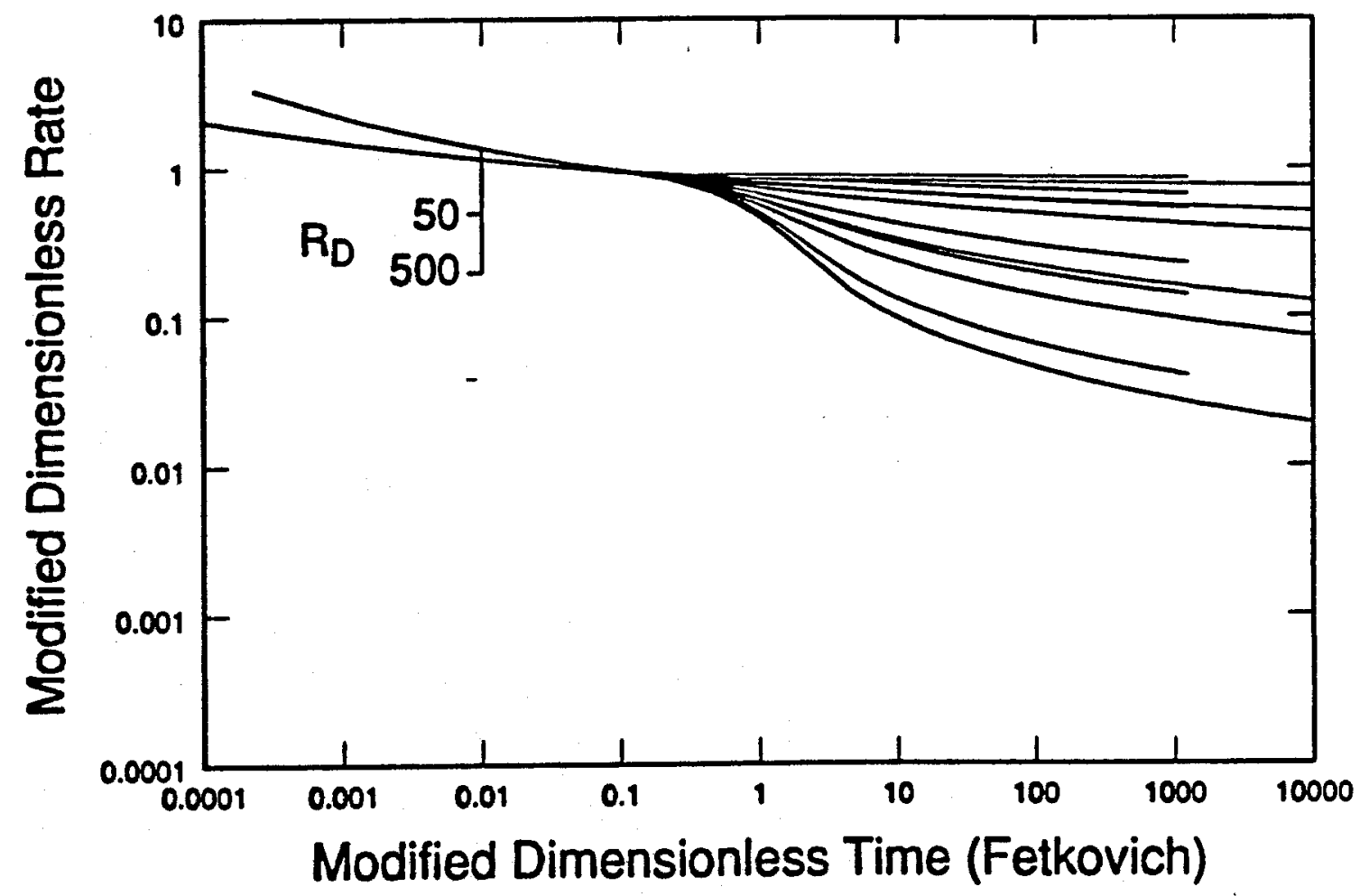

Figure 11. Fetkovich shifted composite reservoir rate declines. Composite reservoir, $\mathrm{R}_{D}=50,500 . \quad \lambda=0.1,0.5,1.0,5.0,10.0,50.0$. 


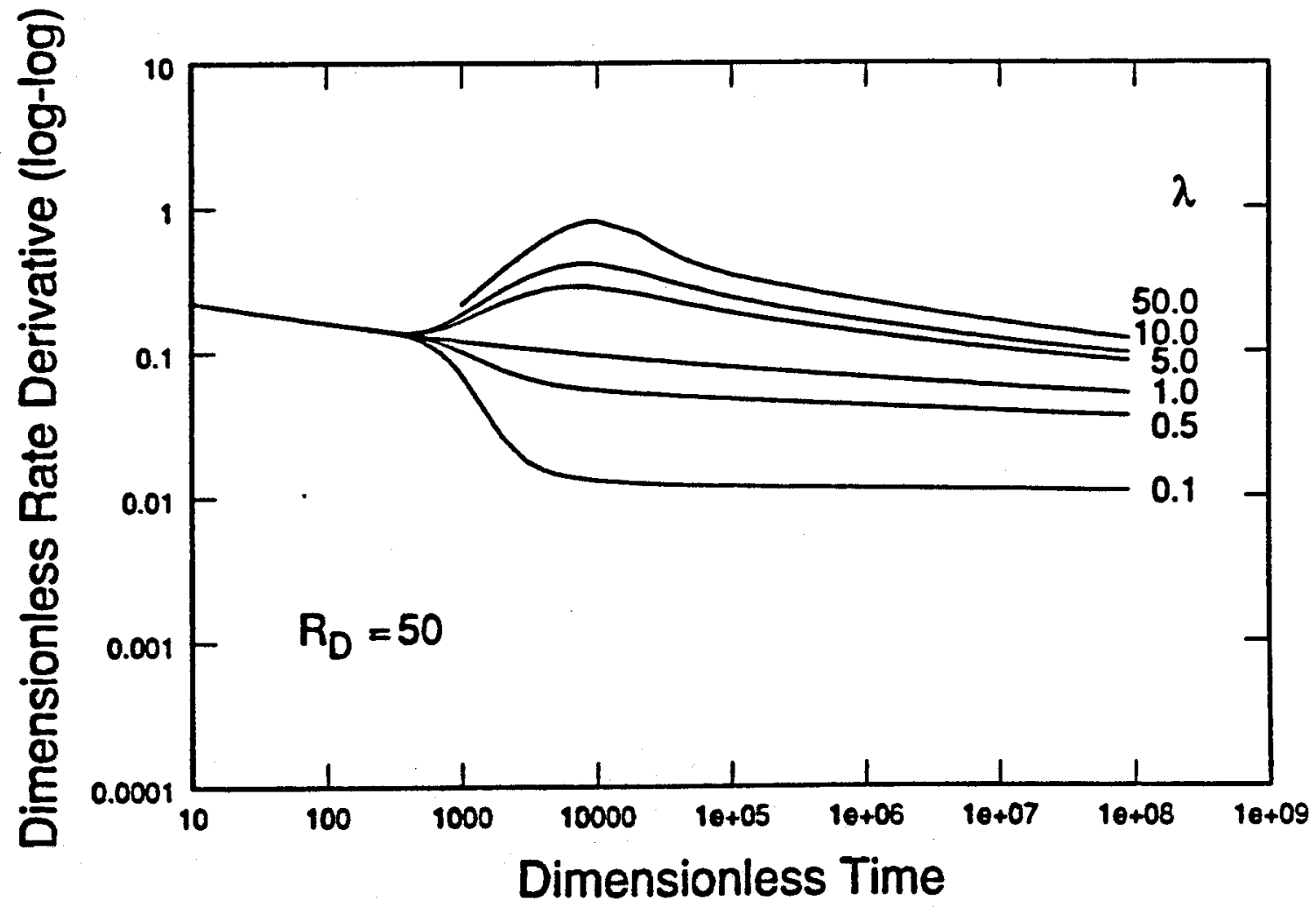

Figure 12. Effects of mobility ratio on $\log -\log$ rate derivatives. Composite reservoir, $R_{D}=50 . \lambda=0.1,0.5,1.0,5.0,10.0,50.0$. 


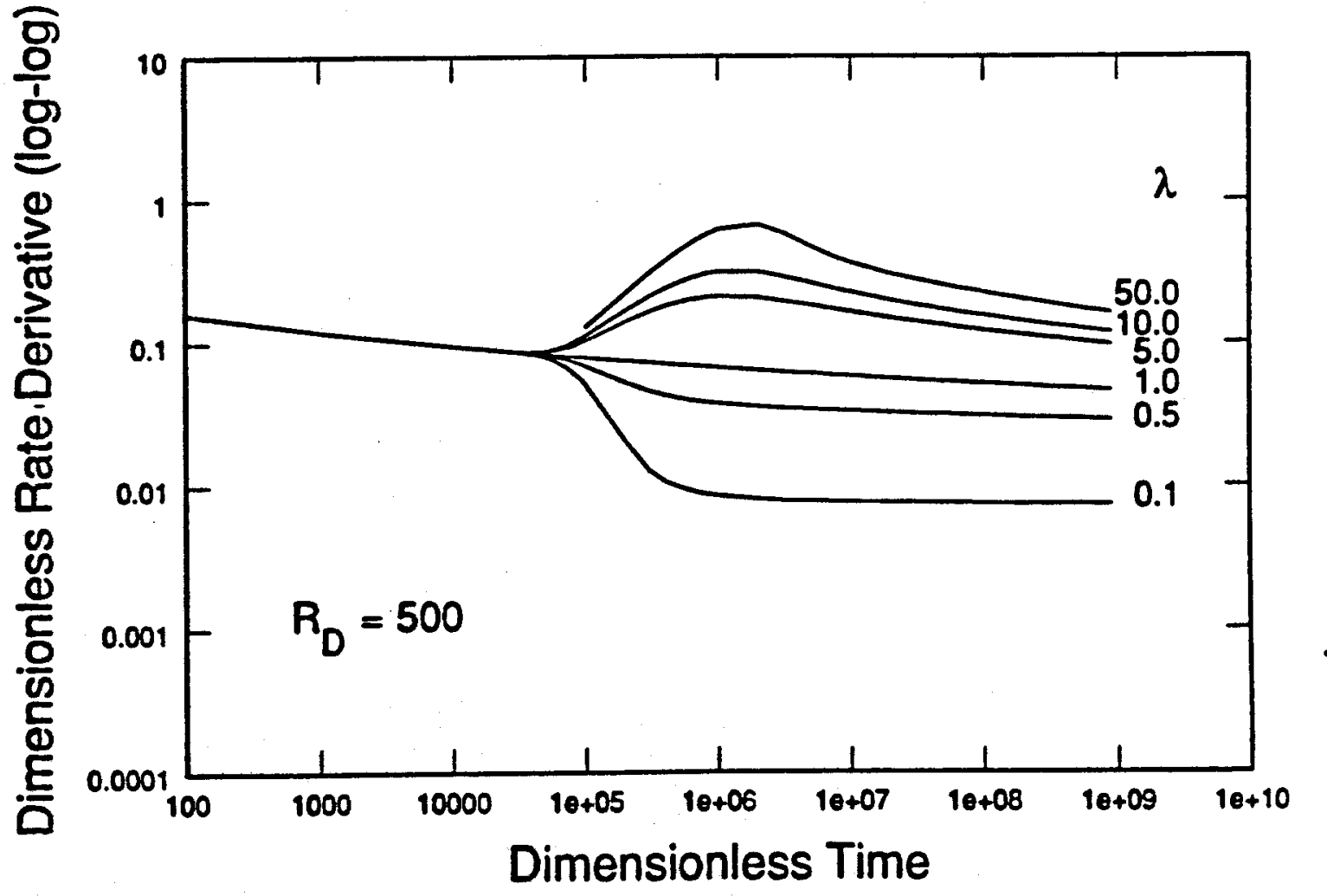

Figure 13. Effects of mobility ratio on $\log -\log$ rate derivatives. Composite reservoir, $R_{D}=500 . \lambda=0.1,0.5,1.0,5.0,10.0,50.0$. 


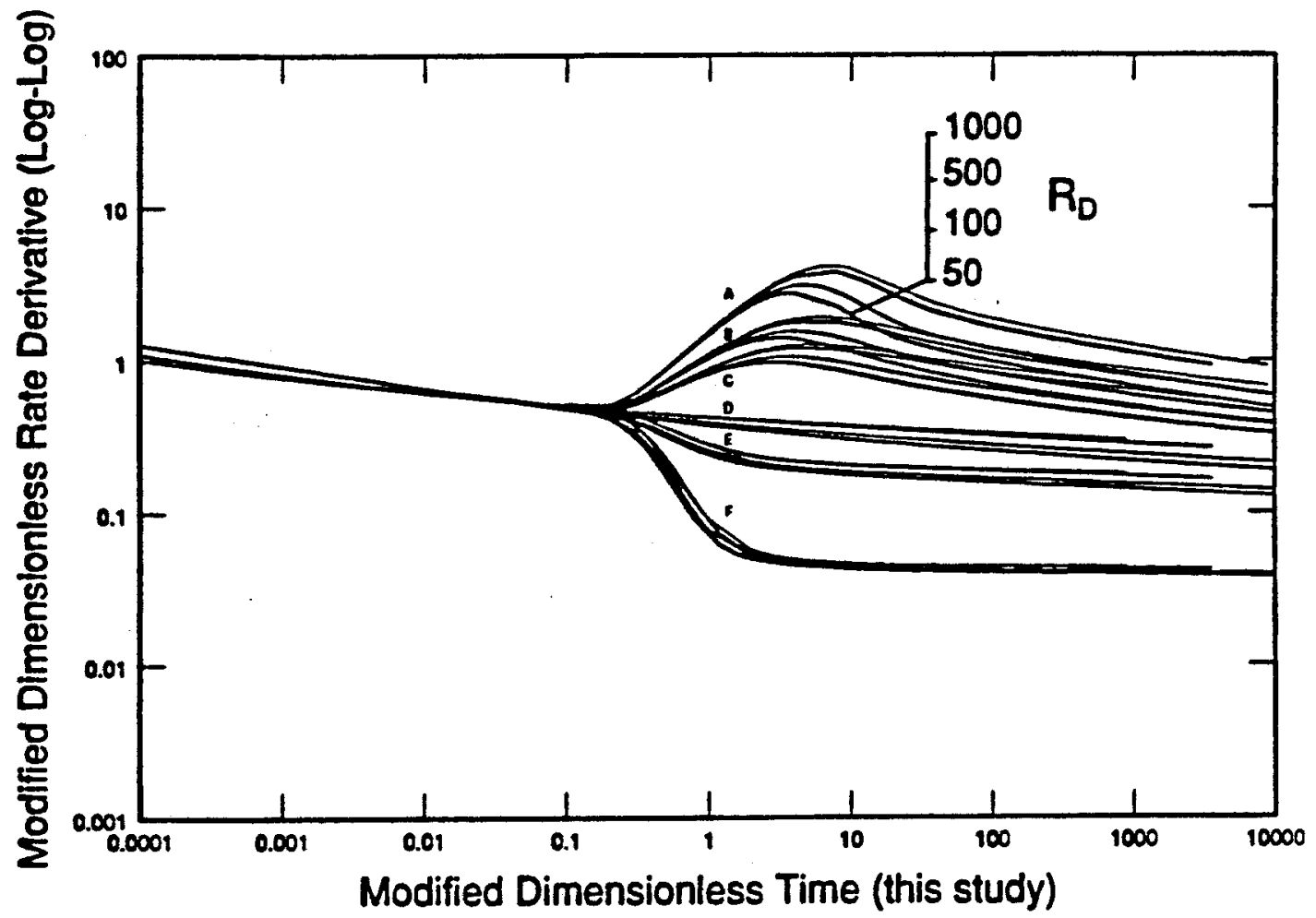

Figure 14. Shifted (this study) composite reservoir $\log -\log$ rate derivatives. $R_{D}=50,100$, 500, 1000. A: $\lambda=50.0$, B: $\lambda=10.0, \mathrm{C}: \lambda=5.0, \mathrm{D}: \lambda=1.0, \mathrm{E}: \lambda=0.5, \mathrm{~F}: \lambda=0.1$. 


\section{ANALYSIS PROCEDURE}

Simulated rate decline data for an infinite composite reservoir with $R_{D}=500$ are graphed in Figure 15. Covering just over three log cycles, the data matches very well over the portion of the curve representing the infinite acting rate response. However, very little character is displayed by this plot; no evidence of exponential decline has appeared yet. This is a problem that is encountered with type curve matching on the Fetkovich collapsed decline curves. If there is not enough data, the radius of the system cannot be determined, and only a minimum radius can be estimated.

If a closed outer boundary is present, or if the reservoir is a composite reservoir. the values of $r_{e D}$ or $R_{D}$ can still be determined with this data. The data for the same case is differentiated and graphed on the derivative response type curve in Figure 16. Here, the derivative response due to the effects of the mobility ratio or a closed outer boundary occurs almost one log cycle earlier than the rate response. While no significant character was evident in the rate response curve, very apparent character is displayed here. The deviation from the infinite acting case is obvious; but it is not known whether it is due to the effects of an unfavorable mobility ratio, or due to the presence of a closed outer boundary. In other words, the value for the minimum $R_{D}$ can be estimated if the conversion factor between $t$ and $t_{D}$ is known. In order to distinguish between these.two, the data would need to extend over the "hump" displayed in the derivative response graph. But the fact remains that the derivative response yields valuable reservoir information almost one log cycle earlier than the corresponding rate response. If $t_{D}$ is known, or if an approximation for $t_{D}$ is available, $R_{D}$ can be estimated from a time match point using equation (61).

The maximum slope obtainable in the derivative response curve is unity. The deviations from unity seen in the composite reservoir derivative response for 


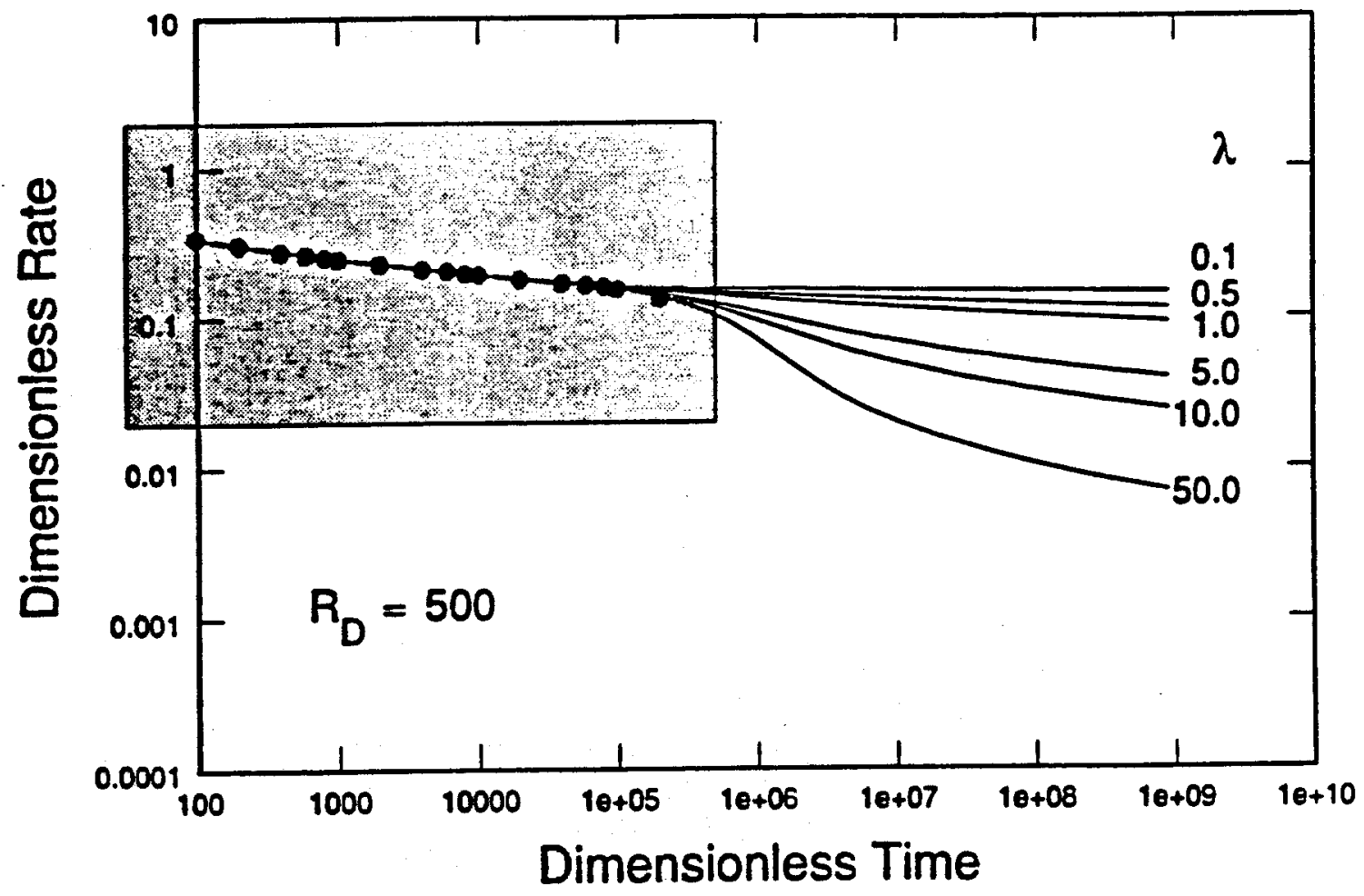

Figure 15. Log-log match of rate data for a simulated example. 


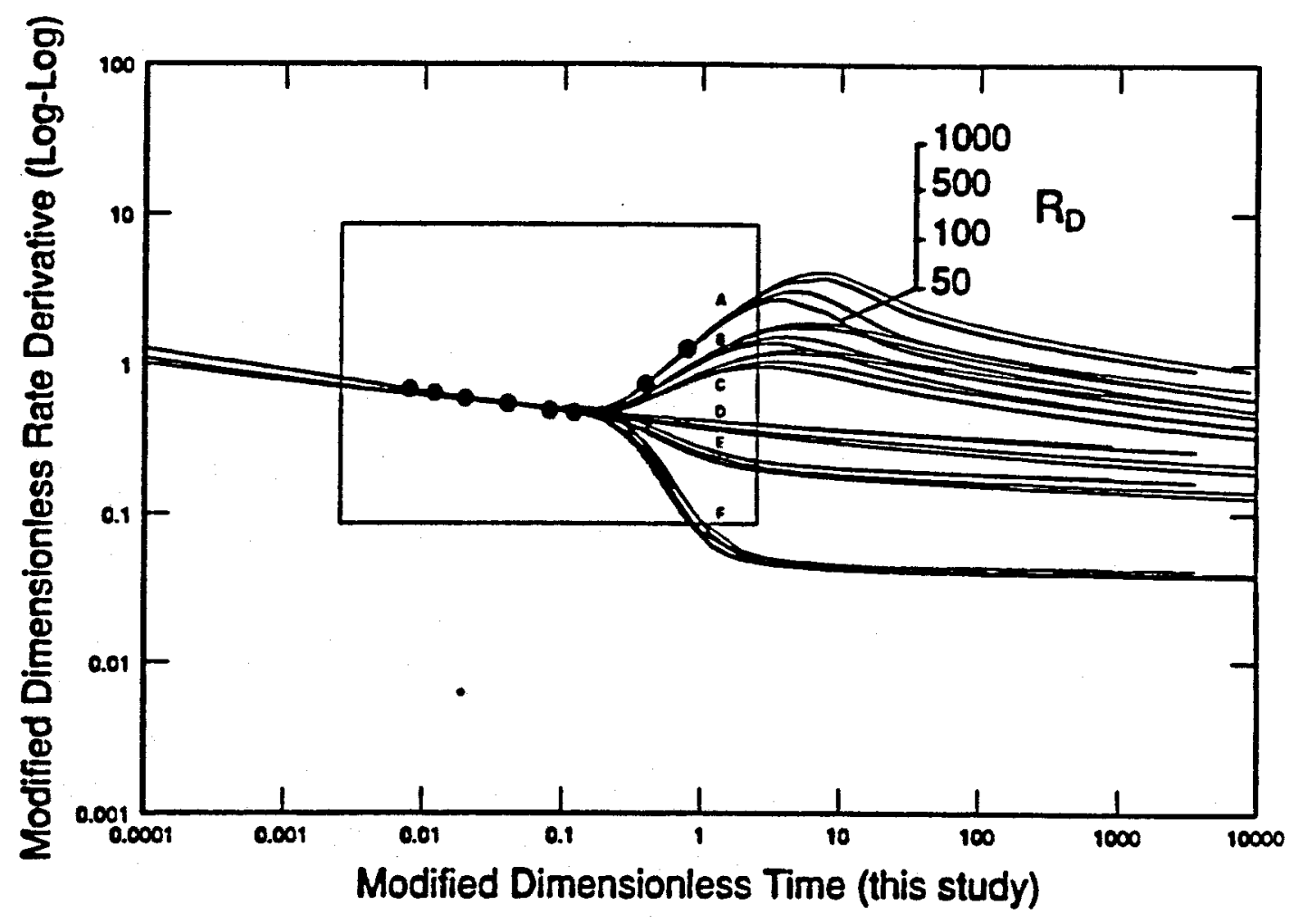

Figure 16. Log-log match of rate derivative data for a simulated example. 
unfavorable mobility ratios is due to the response of the outer reservoir region. Thus, if the derivative data arrives at a slope of unity and remains constant at unity for a long period of time, the response may be due to the effects of a closed outer boundary.

Thus, derivative analysis allows for the detection of a reservoir outer radius or a boundary between two distinct reservoir regions around the well. 


\section{CONCLUSIONS}

1. Rate derivative responses for closed outer boundary and constant pressure outer boundary homogeneous reservoirs are collapsed into one type curve.

2. A new time correlating group was found that is different from the correlating group presented by Fetkovich (1980).

3. Rate derivative responses for infinite composite reservoirs are collapsed into one type curve in the same manner that was used for the homogeneous reservoir cases.

4. If the conversion factor between $t$ and $t_{D}$ is known, the exterior radius, $r_{e D}$, or the distance to the discontinuity, $R_{D}$, can be estimated from a time match point using equation (61).

5. For a composite reservoir case, the match of the derivative data to the type curve may yield the value or a lower limit of the value of the mobility ratio, $\lambda$.

6. The derivative response type curves presented in this study provide valuable reservoir information almost one log cycle earlier than is available from the 


\section{NOMENCLATURE}

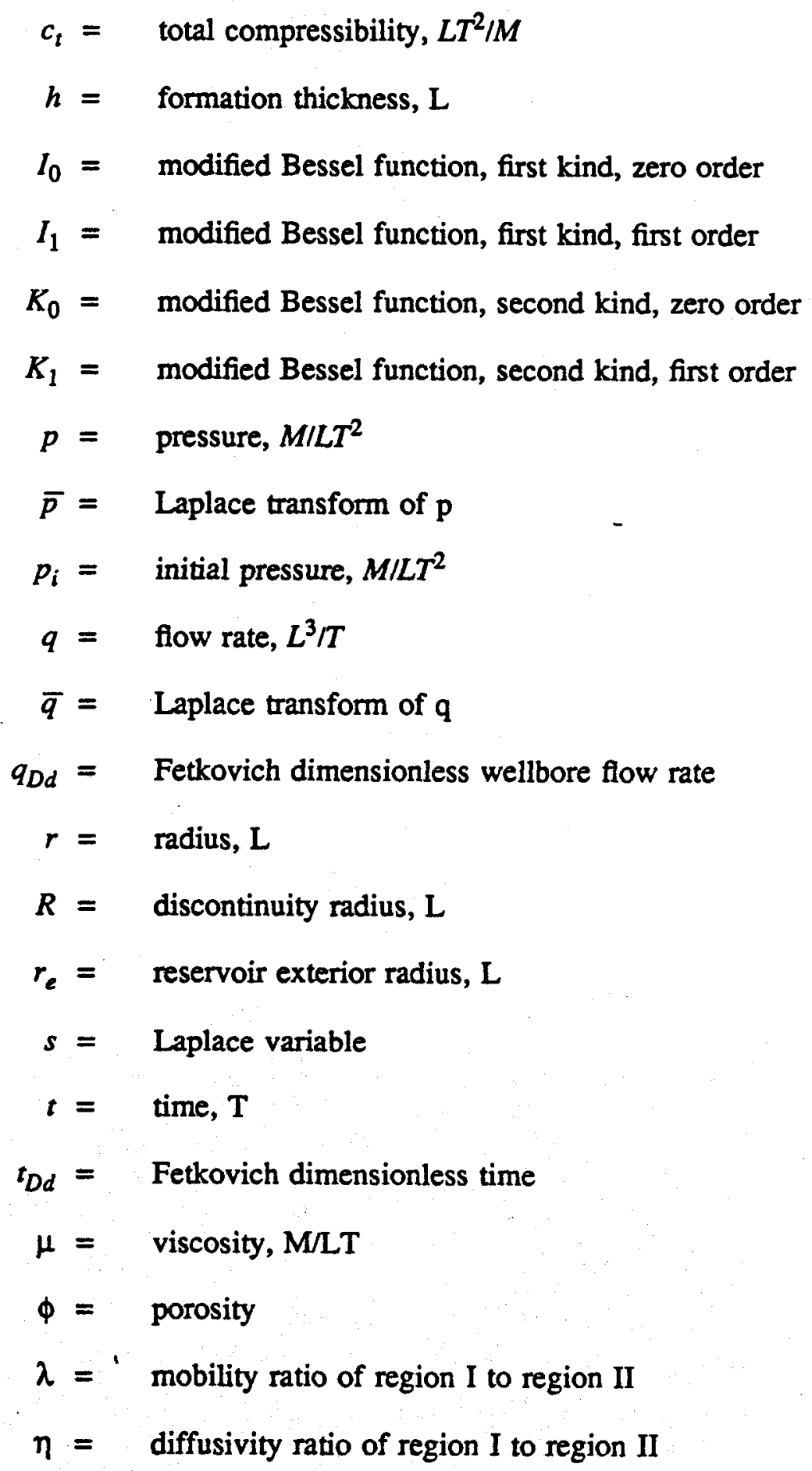




\section{Subscripts}

$$
\begin{aligned}
& 1=\text { region } \mathrm{I} \\
& 2=\text { region } \mathrm{II} \\
& D=\text { dimensionless } \\
& w=\text { wellbore }
\end{aligned}
$$




\section{REFERENCES}

1. Abramowitz, M., and Stegun, I.A.: Handbook of Mathematical Functions, National Bureau of Standards, Washington D. C., 1964.

2. Fetkovich, M.J.: "Decline Curve Analysis Using Type Curves," J. Pet. Tech. (June 1980) 1065-1077.

3. Stehfest, H.: "Algorithm 368, Numerical Inversion of Laplace Transforms," D-5, Communications of ACM, (Jan. 1970), 13, No.1, 49.

4. Turki, L.: "Decline Curve Analysis in Composite Reservoirs," Stanford University, Stanford, California, December, 1986. 